\title{
LIV. On the figure of the earth, as deduced from measurements of different portions of the meridian
}

\author{
J. Ivory M.A. F.R.S.
}

To cite this article: J. Ivory M.A. F.R.S. (1828) LIV. On the figure of the earth, as deduced from measurements of different portions of the meridian , Philosophical Magazine Series 2, 3:17, 343-349, DOI: $10.1080 / 14786442808674661$

To link to this article: http://dx.doi.org/10.1080/14786442808674661

曲 Published online: 10 Jul 2009.

Submit your article to this journal $₫$

Џll Article views: 2

Q View related articles $\llbracket$

47 Citing articles: 1 View citing articles 
of the membrane suddenly ceases almost entirely; but on the fork being turned further, it immediately reappears. If we distinguish in the prongs of the tuning-fork three different surfaces; viz. 1. front and back surfaces; 2 . side surfaces; 3. end surfaces; we observe this disappearance of sound at every transition from a front surface to a side one, and vice versẩ: again, in every transition from a front surface to an end one, and vice versá ; but no cessation of sound is perceived in the transition from a side surface to an end one, and vice versâ. These phænomena are not distinctly audible when there is any noise, for which reason the experiments will be best made at night.

The following differences in these phænomena from those mentioned in the treatise alluded to (in the Jahrbuch 1826, iii. p. 385) are remarkable: 1 . That the membrane is a plane extending far beyond the tuning-fork, and that every point of it can be agitated with equal facility, whilst the reciprocating inclosed column of air had only one narrow opening, and could only resound when the undulations of sound penetrated through this aperture; 2. That the membrane did not reciprocate, and nevertheless showed these phænomena, whilst with a column of air not reciprocating, at least if it be narrow, nothing can be observed.

LIV. On the Figure of the Earth, as deduced from Measurements of different Portions of the Meridian. By J. Ivory, M.A. F.R.S.*

HAVING examined the figure of the earth as deduced from 1 experiments with the pendulum, we are naturally led to consider the same question as it depends upon the measurements that have been made, of different portions of the meridian. If this interesting inquiry be placed within the reach of the human understanding, and be accessible to our industry, the two views of it must be consistent; and, by instituting a comparison between them, we may both learn what is already sufficiently well ascertained, and be directed in our future attempts to the most important points that still remain uncertain.

Let $a$ denote the equatorial radius of the earth; $e$ the eccentricity of the elliptical meridian; $\phi$ an arc of the meridian reckoned from the equator; and $\lambda$ the latitude of the extremity of $\phi$ : then

$$
d \varphi=\frac{a\left(1-e^{2}\right) d \lambda}{\left(1-e^{2} \sin ^{2} \lambda\right)^{\frac{3}{2}}} .
$$

- Communicated by the Author. 


\section{Mr. Ivory on the Figure of the Earth, as deduced from}

If we now put $\sqrt{1-e^{y}}=1-\varepsilon$, then $\varepsilon$ will be the compression or the ellipticity; and we shall have,

$$
d \phi=\frac{a(1-s)^{2} \cdot d \lambda}{\left(1-\left(2 s-\varepsilon^{2}\right) \sin ^{2} \lambda\right)^{\frac{3}{2}}} .
$$

By expanding this formula and integrating as usual, rejecting the cube and higher powers of $\varepsilon$, we shall get,

$$
\varphi=a \cdot\left\{\lambda-\varepsilon\left(\frac{\lambda}{2}+\frac{3}{4} \sin 2 \lambda\right)+\varepsilon^{2}\left(\frac{\lambda}{16}+\frac{15}{64} \sin 4 \lambda\right)\right\} .
$$

And, if $\phi$ denote an arc of the meridian between the latitudes $\lambda^{\prime}$ and $\lambda$; then

$$
\begin{array}{r}
\varphi=a \cdot\left\{\lambda-\lambda^{\prime}-\varepsilon\left(\frac{\lambda-\lambda^{\prime}}{2}+\frac{3}{4}\left(\sin 2 \lambda-\sin 2 \lambda^{\prime}\right)\right)\right. \\
\left.+\varepsilon^{2}\left(\frac{\lambda-\lambda^{\prime}}{16}+\frac{15}{64}\left(\sin 4 \lambda-\sin 4 \lambda^{\prime}\right)\right)\right\} .
\end{array}
$$

Further, let $n=\lambda-\lambda^{\prime}$

$$
\begin{aligned}
m & =\lambda+\lambda^{\prime} \\
p & =57^{0.2957795} \\
\Delta & =\frac{a}{p} ;
\end{aligned}
$$

then, $\phi=\Delta \cdot\left\{n-\varepsilon\left(\frac{n}{2}+\frac{3}{2} p \sin n \cos m\right)\right.$

$$
\left.+\varepsilon^{2}\left(\frac{n}{16}+\frac{15}{16} p \sin n \cos n \cos 2 m\right)\right\} \text {. }
$$

In this formula $\Delta$ is the length of $1^{\circ}$ on the earth's equator; and $n$ and the coefficients of $\varepsilon$ and $\varepsilon^{2}$, are reckoned in degrees.

There has been so much discussion about the merits of the different portions of the meridian that have been determined trigonometrically, and their character is so well known, that it would be superfluous to say any thing on that subject here. The following table, which is taken from a paper by Professor Airy, in the Phil. Trans. for 1826, contains the five arcs which unquestionably deserve the preference, both as they are the longest that have been measured, and likewise on account of the excellent instruments employed, and the great care taken in executing all the operations. But even of these there is one, namely the Swedish arc, measured by Svuanberg, to which some objections are made; because it makes the length of a degree in latitude $66^{\circ} 20^{\prime}$, more than 200 toises less than it had been found to be by Maupertuis and the French Academicians who accompanied him. I shall therefore leave out this arc in determining the elements of the elliptical figure of the earth ; but, these elements being found by means of the other four arcs, we may then apply them to the case omitted, 
Measurements of different Portions of the Meridian. 345 both as some test of their own exactness, and likewise in order to judge what real ground there is for objecting to the Swedish measurement.

\begin{tabular}{|c|c|c|c|}
\hline Country. & $\lambda$ & $\lambda^{\prime}$ & $\begin{array}{c}\phi \\
\text { fathoms. }\end{array}$ \\
\hline Peru. & $-0^{\circ} 2^{\prime} 31^{\prime \prime}$ & $3^{\circ} 4^{\prime} 32^{\prime \prime}$ & 188510 \\
\hline India.... & $\begin{array}{lll}8 & 9 & 38 \cdot 39\end{array}$ & $18 \quad 323 \cdot 6$ & 598630 \\
\hline France........ & $\begin{array}{lll}38 & 39 & 56 \cdot 11\end{array}$ & $\begin{array}{lll}51 & 2 & 9 \cdot 2\end{array}$ & 751567 \\
\hline England...... & $\begin{array}{lll}50 & 37 & 5.27\end{array}$ & $\begin{array}{lll}53 & 27 & 29 \cdot 89\end{array}$ & 172751 \\
\hline Sweden .. & $\begin{array}{lll}65 & 31 & 30 \cdot 27\end{array}$ & $849 \cdot 55$ & 98870 \\
\hline
\end{tabular}

Applying now the data of the four first measurements in the table to the formula (A), the four equations following will be obtained,

$$
\begin{aligned}
& 188510=\Delta\left(3 \cdot 11750-6 \cdot 2261 . \varepsilon+3 \cdot 095 . \varepsilon^{2}\right) \\
& 598630=\Delta\left(9 \cdot 89589-18 \cdot 1985 . \varepsilon+6 \cdot 163 . \varepsilon^{2}\right) \\
& 751567=\Delta\left(12 \cdot 37030-6 \cdot 2811 . \varepsilon-10 \cdot 266 . \varepsilon^{2}\right) \\
& 172751=\Delta\left(2 \cdot 84017-0 \cdot 3844 . \varepsilon-2 \cdot 166 . \varepsilon^{2}\right) .
\end{aligned}
$$

It is known that $\varepsilon$ is not much different from $\frac{1}{30}$, and certainly does not surpass $\frac{1}{5} \overline{\frac{1}{0}}$. We may therefore assume,

$$
\varepsilon=\frac{1}{300}+t ; \quad \varepsilon^{2}=\frac{1}{90000}+\frac{2 t}{300} ;
$$

the correction $t$ being a small fraction which we are sure is less than $\frac{1}{300}$. The foregoing equations will now become, by substitution,

$$
\begin{aligned}
& 188510=\Delta(3.09678-\sigma .206 t) \\
& 598630=\Delta(9.83530-18.157 t) \\
& 751567=\Delta(12.34925-6.350 t) \\
& 172751=\Delta(2.83887-0.399 t) .
\end{aligned}
$$

In order to find a first approximate value of $\Delta$, I add the four equations, neglecting the terms containing $t$ : thus

$$
1711458=28 \cdot 1202 . \Delta ; \Delta=60862 \text {. }
$$

Next, put $\mathrm{D}=60862, \Delta=\mathrm{D}(1+s)$; then, by substituting in the four last equations, we shall get,

$$
\begin{aligned}
\frac{35}{\mathbf{D}} & =3.097 s-6.206 t \\
\frac{35}{\mathbf{D}} & =9.835 s-18.157 t \\
-\frac{33}{\mathbf{D}} & =12.349 s-6.350 t \\
-\frac{27}{\mathrm{D}} & =2.839 s-0.399 t
\end{aligned}
$$

Nere Series. Vol. 3. No. 17. May 1828. 
These equations are next to be reduced to two, the number of the unknown quantities, by employing the method of the least squares : thus

$$
\begin{aligned}
& -\frac{31 \cdot 550}{\mathrm{D}}=267.234 s-277.342 t \\
& +\frac{632.332}{\mathrm{D}}=277.342 s-408.673 t .
\end{aligned}
$$

And, by solving these equations,

$$
\begin{aligned}
\mathrm{D} s & =-6 ; & \Delta=\mathrm{D}+\mathrm{D} s=60856 \\
t & =-.00009, & \varepsilon=\frac{1}{300}+t=\cdot 00324=\frac{1}{309} .
\end{aligned}
$$

Returning now to the original equations (B), and substituting the values of $\Delta$ and $\varepsilon$, we shall obtain the errors, $\mathbf{E}^{(1)}, \mathbf{E}^{(0)}$, $E^{(3)}, E^{(3)}$, or the quantities that must be added to the given measurements on the left sides of the equations, in order to make both sides exactly equal; as follows,

$$
E^{(1)}=-20, E^{2}=+10, E^{(3)}=-7, E^{(3)}=+13 .
$$

The greatest error is in the Peruvian are, which appears to be too great, as has generally been surmised. But the smallness of the errors proves the consistency of the different measurements; and shows that the four portions of the meridian, although very remote from one another, belong to one and the same elliptical spheroid.

If we now substitute the data of the Swedish measurement in the formula $(A)$, we shall get,

$$
98870+\mathrm{E}^{(5)}=\Delta\left(1.622022+0.8378 . \varepsilon-0.022 . \varepsilon^{2}\right):
$$

and, by substituting the values of $\Delta$ and $\varepsilon$, it will appear that the error $E^{(j)}=+5$. This measurement is therefore very consistent with the other four; and, as it seems liable to no just objection, it adds to the evidence in favour of the elliptical elements that have been found.

The degree of the meridian bisected by the parallel of $45^{\circ}$, is 111115 metres at zero of the thermometer. This result is deduced from the actual measurement of the meridional arc between the parallels of Greenwich and Formentera, and is independent of any assumption about the figure of the earth. To reduce this length to fathoms at the temperature of $62^{\circ}$ of Fahr., it must be multiplied by $\frac{39 \cdot 37079}{72}$, which gives 60758 fathoms. Now, if we put $\lambda=45^{\circ}+\frac{1}{2}, \lambda^{\prime}=45-\frac{1}{2}$, we shall have, in he formula (A), $n=1^{\circ}, m=90^{\circ}$, and the length of the degree having its middle point in latitude $45^{\circ}$, will be,

$$
\Delta\left(1-\frac{\varepsilon}{2}-\frac{7}{8} \varepsilon^{2}\right) \text {; }
$$


or, in numbers $60856(1-\cdot 001629)=60757$ fathoms, which is only one fathom less than the observed quantity.

There is also another measurement with which we may compare the elliptical elements that have been found. A portion of the parallel to the equator in latitude $45^{\circ} 43^{\prime} 12^{\prime \prime}$, little short of $13^{\circ}$ in amplitude, has been measured, and the most probable length of $1^{\circ}$ of longitude on that parallel has been fixed at $77865^{\mathrm{m}} \cdot 75^{*}$. The large arc was measured in six portions, the difference of longitude between the extremities of every portion being ascertained by means of signals made by exploding gunpowder. If we compare the six degrees, deduced from the several partial arcs, it must be allowed that their differences are great and irregulart. There must therefore be considerable uncertainty in the arithmetical mean of such irregular quantities; which mean is only three metres less than the most probable value of a degree. The length of a degree deduced from the four partial arcs contained within the territory of France, which together exceed $7^{\circ}$ of longitude, is $77885^{\mathrm{m} \cdot 75}$, or 20 metres more than the length deduced from the whole arc of about $\left.13^{\circ}\right\rfloor$. It is to be observed that the errors of longitude at the intermediate stations accumulate as the arc is extended; so that there is not the same advantage in measuring a large arc of the parallel, as in the case of the meridian; for, in one instance, the total amplitude is affected by the sum of the errors at all the intermediate stations; whereas, in the other, it is affected only by the errors of the two extreme latitudes. If we further add that a great length of the parallel answers to a minute portion of time; in so much that an error of a single second in the amplitude of the large arc, would produce a variation of no small magnitude in the mean degree of longitude; it must be evident that in point of accuracy, we cannot repose the same confidence in a degree of the parallel measured in the manner described, as we can in a degree of the meridian deduced from an arc of nearly the same amplitude.

The radius of the parallel to the equator at the latitude $\lambda$, or the perpendicular drawn to the polar axis, will be found, by the properties of the ellipse, equal to

$$
\frac{a \cos \lambda}{\sqrt{1-e^{2} \sin ^{2} \lambda}}=\frac{a \cos \lambda}{\sqrt{1-\left(2 \varepsilon-\varepsilon^{2}\right) \sin ^{2} \lambda}} \text { : }
$$

And if this expression be expanded and multiplied by $\frac{1}{p}$, we shall have the length of a degree of the parallel equal to

$$
\begin{aligned}
& \Delta \cos \lambda\left\{1+\varepsilon \sin ^{2} \lambda+s^{2}\left(\frac{3 \sin 4 \lambda}{2}-\frac{\sin ^{2} \lambda}{2}\right)\right\} \text {. }
\end{aligned}
$$

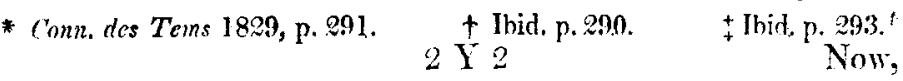


Now, put $\lambda=45^{\circ} 43^{\prime} 12^{\prime \prime}$, and a degree of longitude at that latitude, will be equal

$$
42487.8 \times(1+.001661)=42557 \cdot 4 \text { fathoms. }
$$

But $77865^{\mathrm{m}} \cdot 75$ reduced to fathoms at $62^{\circ}$ of Fahr. is equal to 42578.2 fathoms, which is 20 fathoms more than the calculated quantity. If this discrepancy appear very great, we shall only remark, that it is very small in comparison of the differences of the degrees deduced from the partial arcs, and that it supposes not quite so much as $1^{1 / \frac{1}{2}}$ of time, for the sum of all the accumulated errors in the amplitude of the large arc. Unless we possessed some means of estimating with tolerable exactness the error really existing in the degree of the parallel, the discrepancy we have found can hardly be deemed sufficient to throw any doubt upon the elliptical elements which agree so well with all the most exact measurements that have been made on the earth's surface.

If the earth be really an elliptical spheroid, there can be little doubt that the ellipticity must be very nearly what we have found it to be. But the solution of the problem would be greatly improved, and the results obtained would be more exact and more certain, if the measurement of the meridian in England were extended as far north as possible. An arc of $8^{\circ}$ or $10^{\circ}$ north of Dunnose, would be the most valuable addition that in the present state of this research can be made to the data for determining the figure of the earth. Another very profitable addition would be the extension of the Indian arc, already the largest we have except the French measurement; and more especially if it could he extended southward nearer the equator. Supposing the difference between $\lambda$ and $\lambda^{\prime}$ to be small, it will readily appear that the coefficient of $\varepsilon$ in the formula (A) will be equal to zero, or so small as to render the term insensible, when

$$
\operatorname{Cos}\left(\lambda+\lambda^{\prime}\right)=-\frac{1}{3} \text {, and } \lambda+\lambda^{\prime}=109^{\circ} 28^{\prime} \text {. }
$$

'Thus if, by means of the formula mentioned, we compute the expression of the length of an arc extending northward from Dunnose about a degree beyond Portsoy, to latitude $58^{\circ} 55^{\prime} 30^{\prime \prime}$, it will be found that the length of this large arc is almost independent of the ellipticity $\varepsilon$, and equal to an arc of the equator. The reason is that, in latitude $54^{\circ} 44^{\prime}$, the radius of curvature of the meridian is equal to the radius of the equator, and the degree of the meridian having its middle point in that latitude is equal to $\Delta$, that is, to a degree of the equator. Thus the length of the portion of the meridian contained between the extreme north and south points of Britain depends chiefly on $\Delta$, and very little on the other ele- 
ment $\mathrm{E}$; at the equator, the compression $\varepsilon$ has greater influence on the length of a meridional arc, than at any other position on the surface of the globe; and it follows that two ares of considerable extent, in the situations mentioned, combined with other good measurements in our possession, must lead to a very exact determination of the elliptical elements of the figure of the earth. One remark it seems important to add; namely, that the ellipticity cannot be determined with any precision by combining different degrees of the meridian measured in England; because the element sought has little influence on the lengths of such degrees. And this observation may be extended to all latitudes within $15^{\circ}$ or $20^{\circ}$ of $54^{\circ} 44^{\prime}$ on either side; within which limits the ellipticity contributes only an inconsiderable share to the length of a meridional arc. And what has now been said will serve to explain the discrepancy of the ellipticities deduced by combining different degrees of the meridian within the boundaries of England and France; and likewise the great variation between the quantities so obtained, and what is found by the comparison of remote degrees. These observations may be illustrated by examining the equations (B). In the English and Swedish measurements, the coefficients of $\varepsilon$ are small, and have opposite signs. The coefficients of the same quantity are nearly equal in the French and Peruvian arcs, although the former has more than four times the length of the latter. In the Indian and French measurements, the coefficients are as 3 to 1 , while the arcs themselves are as 3 to 4 ; and this shows how important it is, for the exact determination of the ellipticity, to have a large arc near the equator.

April 12, 1828.

J. IVory.

LV. Mineralogical and Chemical Examination of the Diopside of Fassa in the Tyrol. By Dr. H. W Ackenrover, Professor of Chemistry at Göttingen*.

A MONG the various new minerals discovered by Bonvoi$A$ sin $\dagger$ during a journey through Piedmont, there were two which this mineralogist considered as distinct species of minerals; and he called them, after the places in which they were found, Mussite and Alalite. According to an account given by Tonnellier $t$, Haiiy found in the crystalline form of these two minerals such a degree of conformity as induced him to

* Communicated by the Author.

+ Journ. de Phys. par Delametheric, tom. Ixii. p. 418, 493.

$\ddagger$ Journ. des Mines, tom, xx. 1.65. 